QCD Evolution Workshop 2014

International Journal of Modern Physics: Conference Series

Vol. 37 (2015) 1560034 (9 pages)

(C) The Authors

DOI: $10.1142 / \mathrm{S} 2010194515600344$

\title{
The Boer-Mulders Transverse Momentum Distribution in the Pion and its Evolution in Lattice QCD
}

\author{
M. Engelhardt \\ Department of Physics, New Mexico State University, \\ Las Cruces, NM 88003, USA \\ engel@nmsu.edu \\ B. Musch, P. Hägler and A. Schäfer \\ Institut für Theoretische Physik, Universität Regensburg, \\ 93040 Regensburg, Germany \\ J. Negele \\ Center for Theoretical Physics, \\ Massachusetts Institute of Technology, Cambridge, MA 02139, USA \\ Published 25 February 2015
}

\begin{abstract}
Starting from a definition of transverse momentum-dependent parton distributions (TMDs) in terms of hadronic matrix elements of a quark bilocal operator containing a staple-shaped gauge link, selected TMD observables can be evaluated within Lattice QCD. A TMD ratio describing the Boer-Mulders effect in the pion is investigated, with a particular emphasis on its evolution as a function of a Collins-Soper-type parameter which quantifies the proximity of the staple-shaped gauge links to the light cone.
\end{abstract}

Keywords: Hadron structure; lattice QCD; deep inelastic scattering.

PACS numbers: 12.38.Gc, 13.60.Hb

\section{Introduction}

Transverse momentum-dependent parton distribution functions ${ }^{1}$ (TMDs) encode information about the distribution of transverse (as well as longitudinal) momentum among partons in a hadron, as extracted from physical processes such as semiinclusive deep inelastic scattering (SIDIS) or the Drell-Yan (DY) process. Isolating hadron structure information in terms of distribution functions of this type requires a factorization framework which allows one to disentangle that information from

This is an Open Access article published by World Scientific Publishing Company. It is distributed under the terms of the Creative Commons Attribution 3.0 (CC-BY) License. Further distribution of this work is permitted, provided the original work is properly cited. 


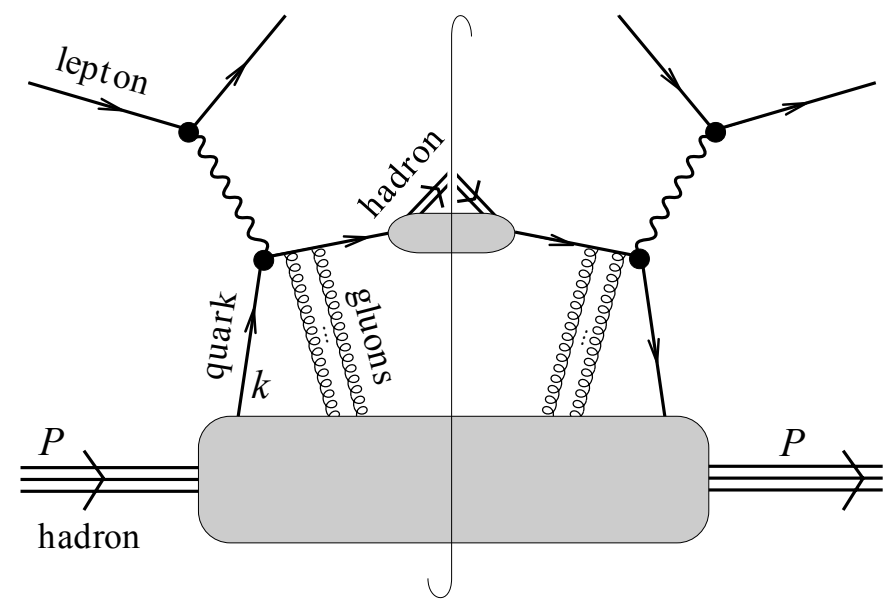

Fig. 1. Illustration of the elements of SIDIS factorization. The lower shaded bubble represents the structure parametrized by TMDs.

other components of the process at hand. At least for selected processes such as the aforementioned, factorization does not seem to face serious obstacles, one possible approach having been advanced in Refs. $[2,3,4]$, although potential issues regarding the analysis of azimuthal asymmetries have recently been noted. ${ }^{5}$

A schematic illustration of the principal elements involved in a description of SIDIS is given by Fig. 1; they include the hard, perturbative vertex, a TMD encoding the structure of the nucleon, and a fragmentation function describing the hadronization of the struck quark. It is important to note that factorization does not necessarily imply that TMDs can be defined completely independent of the process in which they are embedded. In particular, as also indicated in Fig. 1, final-state gluon exchanges between the struck quark and the hadron remnant decisively influence the description of SIDIS (in the DY process, initial-state interactions play an analogous role). Including these final-state interactions also modifies the momentum distributions encoded in the TMDs; the manner in which they formally enter the theoretical definition of TMDs will be elucidated further below.

These final state effects are important in that they break time-reversal invariance and thus generate nontrivial T-odd TMDs, leading to corresponding angular asymmetries in experimental cross sections. Signatures of this kind have indeed been observed experimentally. ${ }^{6}$ Nevertheless, despite the process dependence introduced by accounting for such effects, a "modified universality" across different process types can be retained in a suitable factorization scheme. Specifically, SIDIS and DY can be parametrized by the same TMDs, up to a change in sign in T-odd TMDs. ${ }^{7}$

The project presented here focuses on connecting the phenomenological definition of TMDs, introduced below, to a concrete lattice QCD calculational scheme, 
on the basis of which selected TMD observables are evaluated from first principles. Exploratory results for the T-odd Boer-Mulders transverse momentum shift in the pion are given, expanding on the initial study of nucleon TMDs reported in Ref. [8].

\section{Definition of TMD Observables}

The fundamental correlator defining quark TMDs is of the form

$$
\begin{aligned}
\Phi^{[\Gamma]}\left(x, k_{T}, P, S, \ldots\right)= & \int \frac{d^{2} b_{T}}{(2 \pi)^{2}} \int \frac{d(b \cdot P)}{(2 \pi) P^{+}} \exp \left(i x(b \cdot P)-i b_{T} \cdot k_{T}\right) \cdot \\
& \left.\cdot \frac{\widetilde{\Phi}_{\text {unsubtr. }}^{[\Gamma]}(b, P, S, \ldots)}{\widetilde{\mathcal{S}}\left(b_{T}^{2}, \ldots\right)}\right|_{b^{+}=0}
\end{aligned}
$$

with

$$
\widetilde{\Phi}_{\text {unsubtr. }}^{[\Gamma]}(b, P, S, \ldots) \equiv \frac{1}{2}\langle P, S|\bar{q}(0) \Gamma \mathcal{U}[0, \ldots, b] q(b)| P, S\rangle
$$

The standard phenomenological description employs a Lorentz frame in which the hadron of mass $m_{h}$ propagates with a large momentum in 3-direction, $P^{+} \equiv$ $\left(P^{0}+P^{3}\right) / \sqrt{2} \gg m_{h}$; then, the quark momentum components scale such that the correlator (1) and TMDs derived from it are principally functions of the quark longitudinal momentum fraction $x=k^{+} / P^{+}$and the quark transverse momentum vector $k_{T}$, with the dependence on the component $k^{-} \equiv\left(k^{0}-k^{3}\right) / \sqrt{2} \ll m_{h}$ becoming ignorable in this limit. Correspondingly, (1) is regarded as having been integrated over $k^{-}$; thus, in the Fourier transform, the conjugate component $b^{+}$is set to zero, as written. The hadron momentum and spin are denoted by $P$ and $S$, and $\Gamma$ stands for an arbitrary $\gamma$-matrix structure. The ellipsis in $\Phi^{[\Gamma]}\left(x, k_{T}, P, S, \ldots\right)$ indicates that the correlator will depend on various further parameters, related, e.g., to regularization, specified below as needed. Heuristically, one can view the Fourier-transformed bilocal quark bilinear operator as counting quarks of momentum $k$ in the hadron state, with $\Gamma$ controlling the spinor components involved. However, gauge invariance additionally enforces the introduction of a gauge link $\mathcal{U}$, the precise path of which will be specified presently. The presence of $\mathcal{U}$ introduces divergences additional to the field renormalizations of the quark operators (this is indicated by the subscript "unsubtr."); these divergences accordingly must be compensated by the additional "soft factor" $\widetilde{\mathcal{S}}$. Here, $\widetilde{\mathcal{S}}$ will not need to be specified in detail, since only appropriate ratios in which the soft factors cancel will ultimately be considered.

The gauge link structure $\mathcal{U}$ plays a natural role in the correlator (2), providing a vehicle for incorporating the final state gluon exchanges between struck quark and hadron remnant discussed in connection with Fig. 1. An effective resummation of these interactions yields a Wilson line which approximately follows the trajectory of the struck quark, close to the light cone. The correlator (2), representing the squared amplitude of the physical process, thus has parallel Wilson lines attached to both of the quark operators, extending to large distances along a direction $v$ close to the light cone; at the far end, these lines are connected by a Wilson line in the $b$ 
direction to maintain gauge invariance. The result is the staple-shaped connection $\mathcal{U}[0, \eta v, \eta v+b, b]$, where the path links the positions in the argument of $\mathcal{U}$ with straight line segments, and $\eta$ parametrizes the length of the staple. Formally, it is the introduction of the additional vector $v$ which breaks the symmetry under time reversal and thus generates T-odd TMDs. At first sight, the most convenient choice for the staple direction $v$ would seem to be a light-like vector. However, beyond tree level, this introduces rapidity divergences which require regularization. One advantageous way to accomplish this is to take $v$ slightly off the light cone into the space-like region, ${ }^{2,3}$ with perturbative evolution equations governing the approach to the light cone. ${ }^{4}$ This scheme features the modified universality alluded to further above; the SIDIS and DY processes are connected by inversion of $v$, inducing the proper sign change in T-odd TMDs. A scheme in which $v$ (along with the quark operator separation $b$ ) is generically space-like is also attractive as a starting point for the development of the lattice QCD calculation, as will be discussed below. A useful parameter characterizing how close $v$ is to the light cone is the CollinsSoper evolution parameter $\hat{\zeta}=v \cdot P /(|v||P|)$, in terms of which the light cone is approached for $\hat{\zeta} \rightarrow \infty$.

Decomposing the correlator $\Phi^{[\Gamma]}\left(x, k_{T}, P, S, \ldots\right)$ into the relevant Lorentz structures yields the TMDs as coefficient functions. Whereas in the nucleon case, this leads to eight distinct leading-twist TMDs, in the simpler $S=0$ pion case investigated here, only two leading-twist TMDs remain, namely, the unpolarized TMD $f_{1}$ and the T-odd Boer-Mulders TMD $h_{1}^{\perp}$, given, respectively, by

$$
\Phi^{\left[\gamma^{+}\right]}=f_{1} \quad, \quad \Phi^{\left[i \sigma^{i+} \gamma^{5}\right]}=\frac{\epsilon_{i j} k_{j}}{m_{\pi}} h_{1}^{\perp}
$$

The latter characterizes the distribution of transversely polarized quarks in the (unpolarized) pion.

On the other hand, also the position space correlator $\widetilde{\Phi}_{\text {unsubtr. }}^{[\Gamma]}$, cf. $(2)$, which represents the quantity amenable to lattice evaluation, can be decomposed analogously in terms of invariant amplitudes $\widetilde{A}_{i B}$. Again, for $S=0$, only two amplitudes from the full set obtained for non-zero $\operatorname{spin}^{8}$ remain,

$$
\frac{1}{2 P^{+}} \widetilde{\Phi}_{\text {unsubtr. }}^{\left[\gamma^{+}\right]}=\widetilde{A}_{2 B} \quad, \quad \frac{1}{2 P^{+}} \widetilde{\Phi}_{\text {unsubtr. }}^{\left[i \sigma^{i+} \gamma^{5}\right]}=i m_{\pi} \epsilon_{i j} b_{j} \widetilde{A}_{4 B}
$$

These amplitudes are useful in that they can be evaluated in any desired Lorentz frame, including one particularly suited for the lattice calculation. In view of (3), they are clearly closely related to Fourier-transformed TMDs. Performing the corresponding algebra, and specializing, for the purposes of the present investigation, to the lowest $x$-moment by choosing $b \cdot P=0$, one has

$$
\begin{aligned}
\tilde{f}_{1}^{[1](0)}\left(b_{T}^{2}, \hat{\zeta}, \ldots, \eta v \cdot P\right) & =2 \widetilde{A}_{2 B}\left(-b_{T}^{2}, b \cdot P=0, \hat{\zeta}, \eta v \cdot P\right) / \widetilde{\mathcal{S}}\left(b_{T}^{2}, \ldots\right) \\
\tilde{h}_{1}^{\perp[1](1)}\left(b_{T}^{2}, \hat{\zeta}, \ldots, \eta v \cdot P\right) & =2 \widetilde{A}_{4 B}\left(-b_{T}^{2}, b \cdot P=0, \hat{\zeta}, \eta v \cdot P\right) / \widetilde{\mathcal{S}}\left(b_{T}^{2}, \ldots\right)
\end{aligned}
$$


where the generic Fourier-transformed TMD is defined as ${ }^{9}$

$$
\tilde{f}^{[1](n)}\left(b_{T}^{2}, \ldots\right)=n !\left(-\frac{2}{m_{N}^{2}} \partial_{b_{T}^{2}}\right)^{n} \int_{-1}^{1} d x \int d^{2} k_{T} e^{i b_{T} \cdot k_{T}} f\left(x, k_{T}^{2}, \ldots\right)
$$

The $b_{T} \rightarrow 0$ limit formally yields $k_{T}$-moments of TMDs. However, this limit contains additional singularities, which one can view as being regulated by a finite $b_{T}$. Here, results will only be given at finite $b_{T}$. It is important to note the presence of the soft factors $\widetilde{\mathcal{S}}$ on the right-hand sides of (5) and (6). Absent an evaluation of these soft factors, which themselves depend on $b_{T}^{2}$, one cannot directly Fourier-transform the amplitudes $\widetilde{A}_{i B}$ extracted from a lattice calculation back to momentum space to obtain the original TMDs defined in (3). On the other hand, one can construct an observable in which the soft factors cancel by normalizing the (Fourier-transformed) Boer-Mulders function (6) by the unpolarized TMD (5), which essentially counts the number of valence quarks. Thus, one defines the "generalized Boer-Mulders shift"

$$
\left\langle k_{y}\right\rangle_{U T}\left(b_{T}^{2}, \ldots\right) \equiv m_{\pi} \frac{\tilde{h}_{1}^{\perp[1](1)}\left(b_{T}^{2}, \ldots\right)}{\tilde{f}_{1}^{[1](0)}\left(b_{T}^{2}, \ldots\right)}=m_{\pi} \frac{\widetilde{A}_{4 B}\left(-b_{T}^{2}, 0, \hat{\zeta}, \eta v \cdot P\right)}{\widetilde{A}_{2 B}\left(-b_{T}^{2}, 0, \hat{\zeta}, \eta v \cdot P\right)}
$$

which is the regularized, finite- $b_{T}$ generalization of the "Boer-Mulders shift"

$$
m_{\pi} \frac{\tilde{h}_{1}^{\perp[1](1)}(0, \ldots)}{\tilde{f}_{1}^{[1](0)}(0, \ldots)}=\left.\frac{\int d x \int d^{2} k_{T} k_{y} \Phi^{\left[\gamma^{+}+s^{j} i \sigma^{j+} \gamma^{5}\right]}\left(x, k_{T}, P, \ldots\right)}{\int d x \int d^{2} k_{T} \Phi^{\left[\gamma^{+}+s^{j} i \sigma^{j+} \gamma^{5}\right]}\left(x, k_{T}, P, \ldots\right)}\right|_{s_{T}=(1,0)}
$$

which, in view of the right-hand side, formally represents the average transverse momentum of quarks polarized in the transverse (" $T$ ") direction orthogonal to said momentum in an unpolarized (" $U$ ") pion, normalized to the corresponding number of valence quarks. In the interpretation of (9), it should be noted that the numerator sums over the contributions from quarks and antiquarks, whereas the denominator contains the difference between quark and antiquark contributions, thus giving the number of valence quarks. Note furthermore that ratios of the type (8) also cancel $\Gamma$-independent multiplicative field renormalization constants attached to the quark operators in (2) at finite physical separation $b$.

\section{Lattice Evaluation and Results}

The formal framework laid out above provides all the necessary elements for a lattice QCD evaluation of the generalized shift (8). One evaluates pion matrix elements of the type (2), which yields the relevant invariant amplitudes $\widetilde{A}_{i B}$ via (4). This requires a setting in which the four-vectors $b$ and $v$ are generically space-like, for the following reason: By employing a Euclidean time coordinate to project out hadron ground states via Euclidean time evolution, lattice QCD cannot straightforwardly accomodate operators containing Minkowski time separations. The operator of which one takes matrix elements thus has to be defined at a single time. Only if both $b$ and $v$ are space-like is there no obstacle to boosting the problem to a Lorentz frame in which $b$ and $v$ are purely spatial, and evaluating $\widetilde{\Phi}_{\text {unsubtr. }}^{[\Gamma]}$ in that frame. 
The results extracted for the invariant amplitudes $\widetilde{A}_{i B}$ are then immediately valid also in the original frame in which (2) was initially defined, thus completing the determination of the shift (8).

Since, in a numerical lattice calculation, the staple extent $\eta$ necessarily remains finite, two extrapolations must be performed from the generated data, namely, $\eta \rightarrow \infty$, as well as the extrapolation of the staple direction toward the light cone, $\hat{\zeta} \rightarrow \infty$. In a previous investigation of nucleon TMDs, ${ }^{8}$ the former extrapolation was seen to be under control for a range of parameters, whereas the latter presented a formidable challenge. The main limitation in this respect is the set of hadron momenta $P$ accessible with sufficient statistical accuracy. One of the main motivations for the present pion study was to achieve progress with respect to the large- $\hat{\zeta}$ limit. The pion, by virtue of its lower mass compared to the nucleon (note that the hadron mass enters the denominator of $\hat{\zeta}$ ), allows one to access higher $\hat{\zeta}$; also, being spinless, it allows one to obtain better statistics for the TMD matrix element (2) by averaging over spatial rotations of the operator under consideration. The calculations presented in the following were performed using a MILC 2+1-flavor gauge ensemble ${ }^{10}$ on $20^{3} \times 64$ lattices with a spacing of $a=0.12 \mathrm{fm}$, corresponding to pion mass $m_{\pi}=518 \mathrm{MeV}$. The largest $\hat{\zeta}$ value reached is $\hat{\zeta}=2.03$. Disconnected contributions to the matrix elements $(2)$ were not evaluated.

Fig. 2 shows a typical result for the generalized Boer-Mulders shift (8) for $u$ quarks at given $\left|b_{T}\right|$ and $\hat{\zeta}$ as a function of the staple extent, comparing the result for a $\pi^{+}$meson with the case of a proton studied previously in Ref. [8]. The T-odd behavior of this observable is evident, with $\eta \rightarrow \infty$ corresponding to the SIDIS limit, whereas $\eta \rightarrow-\infty$ yields the DY limit. The data level off to approach clearly identifiable, stable plateaux as the staple length grows. Note that the two sets of data correspond to identical hadron momentum $P$, and the corresponding $\hat{\zeta}$ values differ only because of the hadron mass in the denominator of $\hat{\zeta}$; i.e., $m_{h} \hat{\zeta}$ is the same in the two cases. In this particular juxtaposition, the Boer-Mulders shifts
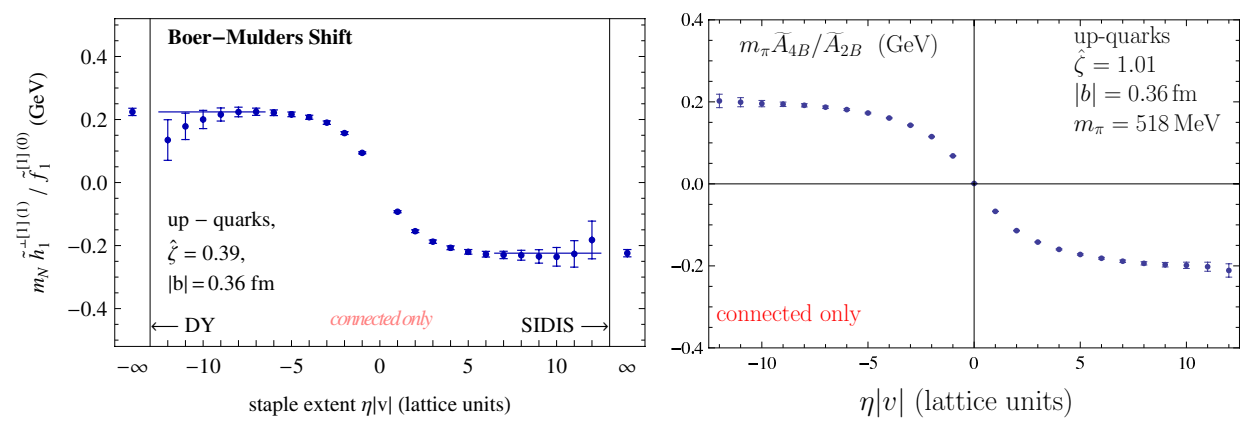

Fig. 2. Generalized Boer-Mulders shift as a function of staple extent for $u$-quarks in a proton (left) and a $\pi^{+}$meson (right). Data are obtained in the same, $m_{\pi}=518 \mathrm{MeV}$, gauge ensemble at identical $\left|b_{T}\right|$ and $m_{h} \hat{\zeta}$. 

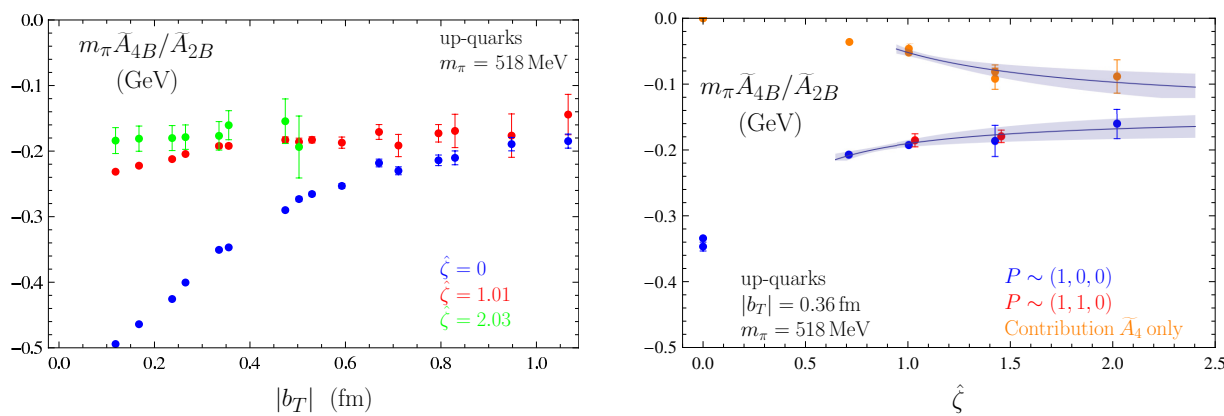

Fig. 3. Generalized Boer-Mulders shift in the $\eta \rightarrow \infty$ SIDIS limit as a function of $\left|b_{T}\right|$ (left) and $\hat{\zeta}$ (right). In the left panel, the data in the region below $\left|b_{T}\right| \approx 0.25 \mathrm{fm}$ may be significantly affected by finite lattice cutoff effects. In the right panel, the congruence of the data obtained for $P$ in different directions exhibits the good rotational properties of the calculation.

are quantitatively very close to one another, in accordance with a suggestion put forward in Ref. [11]. Note, however, that this observation is special to $u$-quarks; the $d$-quark Boer-Mulders shift in the proton is significantly stronger than the $u$-quark shift, whereas the $u$-quark and $\bar{d}$-quark shifts in the $\pi^{+}$are, of course, identical.

Figure 3 focuses on the data in the SIDIS limit. The left panel shows the dependence on $b_{T}$ for three different values of the Collins-Soper evolution parameter $\hat{\zeta}$; the dependence flattens as $\hat{\zeta}$ is increased, and the data for different $\hat{\zeta}$ approach each other at large $b_{T}$. It would be useful to understand this behavior in detail. Focusing on a particular value of $b_{T}$, Fig. 3 (right) displays the $\hat{\zeta}$-dependence of both the full Boer-Mulders shift as well as a certain partial contribution to the Boer-Mulders shift (denoted " $\widetilde{A}_{4}$ only") which vanishes at $\hat{\zeta}=0$, but dominates the quantity at large $\hat{\zeta}$; comparison of the full Boer-Mulders shift with the partial contribution thus can give an indication of convergence towards the large $\hat{\zeta}$ limit. For further details, cf. Ref. [8]. Here, the $\widetilde{A}_{4}$ contribution already furnishes roughly one half of the full shift at the highest $\hat{\zeta}$ reached, signaling that the calculation has covered a significant part of the evolution to large $\hat{\zeta}$. Still, the pion momenta employed are too small

Table 1. Extrapolations of $\left|b_{T}\right|=0.36 \mathrm{fm}$ data toward the large $\hat{\zeta}$ limit. In the combined fit, both the full Boer-Mulders shift as well as the partial $\widetilde{A}_{4}$ contribution are fit simultaneously with a common constant $a$ (but, of course, separate coefficients $b$ ). The right-hand column gives a measure of how well the combined fit ansatz matches the data by quoting the RMS deviation from the data points; given that this deviation is less than, or comparable to the statistical fluctuations for both fit ansätze, they both appear viable, but the $a+b / \hat{\zeta}$ form clearly results in a more favorable fit.

\begin{tabular}{ccccc}
\hline Fit function & $\begin{array}{c}\text { Full BM ratio } \\
(\mathrm{GeV})\end{array}$ & $\begin{array}{c}\text { Contribution } \widetilde{A}_{4} \\
\text { only }(\mathrm{GeV})\end{array}$ & $\begin{array}{c}\text { Combined fit } \\
(\mathrm{GeV})\end{array}$ & $\begin{array}{c}\text { RMS deviation of } \\
\text { combined fit }(\mathrm{GeV})\end{array}$ \\
\hline$a+b / \hat{\zeta}$ & $-0.146(26)$ & $-0.141(36)$ & $-0.145(25)$ & 0.00755 \\
$a+b / \hat{\zeta}^{2}$ & $-0.166(16)$ & $-0.110(22)$ & $-0.148(15)$ & 0.01695 \\
\hline
\end{tabular}


to guarantee a reliable connection to perturbative evolution; the functional form $a+b / \hat{\zeta}$ employed in the fits shown in Fig. 3 thus should be regarded as no more than an ad hoc ansatz. Table 1 summarizes the results of these fits as well as analogous fits using the form $a+b / \hat{\zeta}^{2}$. With the former fit form, the asymptotic values of the full Boer-Mulders shift and the partial $\widetilde{A}_{4}$ contribution are compatible with one another and with the result of a combined fit. This accords with expectations and demonstrates that lattice calculations can achieve a signal for the Boer-Mulders shift of sufficient quality such that it survives taking the $\hat{\zeta} \rightarrow \infty$ limit.

\section{Summary}

The present study focused on the Boer-Mulders shift in a pion, with a particular emphasis on obtaining information concerning the behavior of this type of TMD observable for large Collins-Soper parameter $\hat{\zeta}$. The Boer-Mulders shift is determined from pion matrix elements of a quark bilocal operator containing a staple-shaped gauge link which serves to incorporate final/initial state effects (for SIDIS/DY); the connection between the Lorentz frame preferred for the lattice calculation (in which the staple is defined at a single Euclidean time) and the Lorentz frame preferred for the phenomenological definition of TMDs (in which the staple direction approaches the light cone from the space-like side, as parametrized by $\hat{\zeta}$ ) is achieved by extracting invariant amplitudes from the data. The results for the Boer-Mulders shift suggest an early onset of asymptotic behavior as a function of $\hat{\zeta}$; conclusions about the light-cone limit thus appear to be within reach of lattice calculations. Furthermore, the Boer-Mulders shift for $u$-quarks in protons, investigated previously in Ref. [8], and $\pi^{+}$mesons was observed to be quantitatively similar.

\section{Acknowledgments}

The lattice calculations performed in this work relied on the Chroma software suite ${ }^{12}$ and employed computing resources provided by the U.S. DOE through USQCD at Jefferson Lab. Support by the Heisenberg-Fellowship program of the DFG (P.H.), SFB/TRR-55 (A.S.), and the U.S. DOE and the Office of Nuclear Physics through grants DE-FG02-96ER40965 (M.E.) and DE-SC0011090 (J.N.), as well as through contract DE-AC05-06OR23177, under which Jefferson Science Associates, LLC, operates Jefferson Laboratory (B.M.), is acknowledged.

\section{References}

1. D. Boer, M. Diehl, R. Milner, R. Venugopalan, W. Vogelsang et al., arXiv:1108.1713.

2. S. M. Aybat and T. C. Rogers, Phys. Rev. D 83, 114042 (2011).

3. J. C. Collins, Foundations of Perturbative QCD (Cambridge University Press, 2011).

4. S. M. Aybat, J. C. Collins, J.-W. Qiu and T. C. Rogers, Phys. Rev. D 85, 034043 (2012).

5. M. G. A. Buffing and P. J. Mulders, Phys. Rev. Lett. 112, 092002 (2014).

6. A. Airapetian et al., HERMES Collaboration, Phys. Rev. Lett. 103, 152002 (2009). 
7. J. Collins and A. Metz, Phys. Rev. Lett. 93, 252001 (2004).

8. B. Musch, P. Hägler, M. Engelhardt, J. W. Negele and A. Schäfer, Phys. Rev. D 85, 094510 (2012).

9. D. Boer, L. Gamberg, B. Musch and A. Prokudin, JHEP 1110021 (2011).

10. C. Aubin et al., Phys. Rev. D 70, 094505 (2004).

11. M. Burkardt and B. Hannafious, Phys. Lett. B658, 130 (2008).

12. R. G. Edwards and B. Joó, SciDAC Collaboration, Nucl. Phys. Proc. Suppl. 140, 832 (2005). 